\title{
Flexure-Based 6-Axis Alignment Module for Automated Laser Assembly
}

\author{
Christian Brecher, Nicolas Pyschny, and Jan Behrens \\ Fraunhofer Institute for Production Technology IPT, \\ Department for Production Machines, \\ Steinbachstrasse 17, 52074 Aachen, Germany \\ nicolas.pyschny@ipt.fraunhofer.de
}

\begin{abstract}
Fully automating the assembly of laser systems puts high demands on the accuracy, but alike on the flexibility and adaptivity of the assembly system. In this paper a concept for a flexible robot-based precision assembly is introduced. This concept is based on a modular 6-axis alignment tool which has been designed as a hybrid serial-parallel manipulator with flexures for all revolute and spherical joints. Technical insights on the design and dimensioning based on analytical calculations will be presented as well as first results from the characterization of a prototypal alignment module.
\end{abstract}

Keywords: Precision assembly, parallel manipulator, compliant mechanism, flexures.

\section{Introduction}

Laser applications, especially laser marking and engraving, are about to reach the long desired status of a commodity. Thus, the technological developments of optomechanical products are driven by dynamically changing customer demands and miniaturization, posing a major challenge for high precision manufacturing and assembly [1].

Mainly, the assembly of optical systems is a very challenging task due to highest requirements on alignment accuracies and significant influences of component tolerances. Hence, nowadays the assembly is dominated by manual operations, involving elaborate alignment by means of adjustable mountings and the application of multiple sensors. Economically, further automated assembly of high-quality optical systems, such as laser units, could be the way to produce more reliable units at lower cost. Nevertheless, the multi-functional and hybrid character of lasers puts a high demand on assembly systems, which must be able to handle precision assembly and simultaneously cope with an increasing number of product variants.

Presently, a lack of suitable assembly systems with the required precision and flexibility prevents a time and cost efficient automated assembly. The state of the art is characterized by manual and semi-automated solutions with specialized joining technologies for different parts and components [2]. Existing automation solutions for precision assembly of hybrid products are rigid and inflexible and therefore highly 
restricted in their adoption for different assembly tasks. There is a lack of adequate equipment for flexible, automated and high precision gripping, manipulating, positioning, alignment and joining of optical components and systems [3].

\section{Flexibly Automated Assembly of a Miniaturized Solid State Laser}

New solutions for a flexible and automated assembly of hybrid high-precision products - focused on the assembly of a miniaturized diode-pumped solid-state (DPSS) laser for labeling and marking applications - are under development within the major research initiative "Aachen House of Integrative Production" [4]. The miniaturized laser system, designed and developed at the Fraunhofer ILT, is a DPSS laser in planar configuration enabling automated assembly. All optical components can be positioned and assembled from above and are soldered on a ceramic base plate (Figure 1). The approach to automated assembly is based on a thoroughly modular assembly cell, involving three industrial robots, equipped with configurable tools and sensors for handling and joining, machine vision and illumination. The entire system is continuously updated with information about the assembly process, provided from multiple sensors: not only the robot-based camera, but also a camera-based laser beam analysis system and additional sensors for controlling the laser itself.

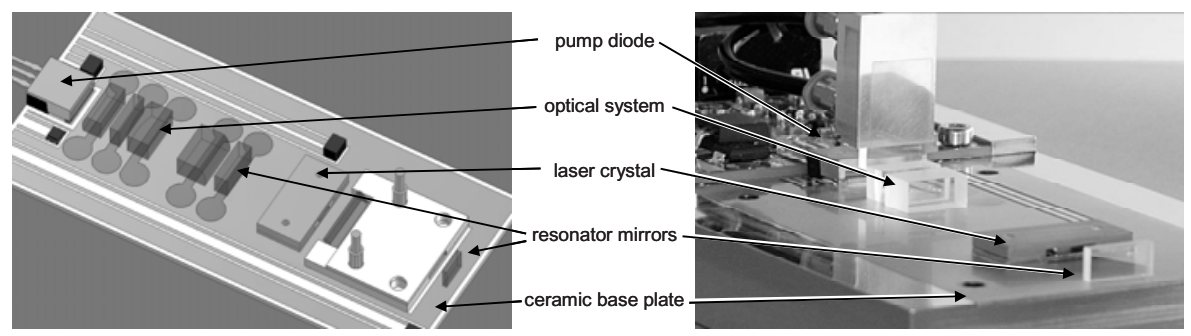

Fig. 1. Miniaturized solid state laser for automated assembly MicroSlab[2]: left shows the model of the optical system (size of the base plate around $40 \mathrm{~mm} \times 100 \mathrm{~mm}$ ), right shows a scene from an assembly sequence

For the alignment of components in the miniaturized solid state laser system a flexible and fast handling system has been developed, consisting of a conventional industrial robot and a high-precision alignment module. The module is mounted to the imprecise but dynamic robot serving as a mobile six-axis alignment tool. It is prepositioned within the large working area of the robot and compensates for position and alignment errors with sub-micrometer accuracy. For the assembly of the optical system and the resonator mirrors an active alignment based on the evaluation of the components' beam transfer functions will be applied (Figure 2). 


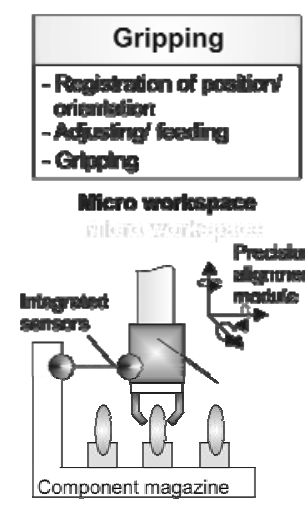

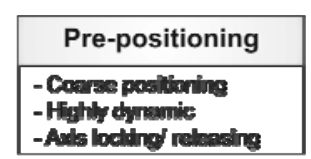

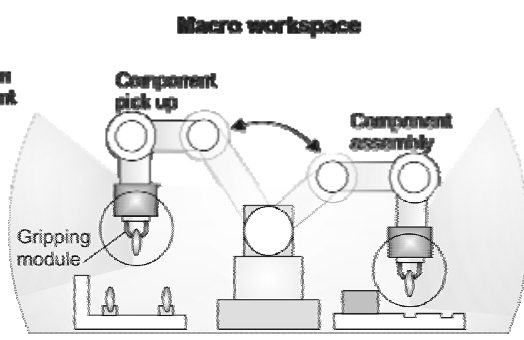

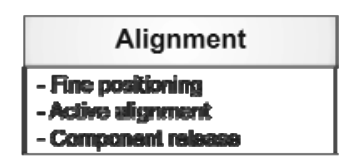

Ritero verispace

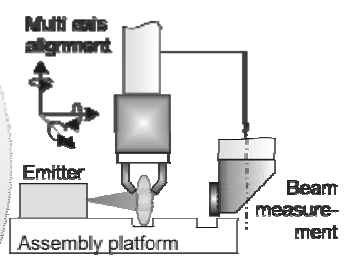

Fig. 2. Alignment concept with modular 6-axis fine-positioning module

A second robot carries modular sensor tools for the evaluation of alignment results, and an innovative soldering technique is being developed and implemented in another robot-based tool to allow for a fully-automated assembly.

\section{Kinematical Analysis and Optimization of Alignment Module}

For the precision manipulation of optical components a miniaturized alignment module with six degrees of freedom (DoF) was to be developed that provides high resolution and high stiffness for robot-based alignment operations. Technical requirements for the laser assembly were an overall weight of less than $1 \mathrm{~kg}$ with outer dimension of about $100 \mathrm{~mm} \times 100 \mathrm{~mm} \times 100 \mathrm{~mm}$, a payload of 200 grams and sub-micrometer resolution in a work-space of at least $1 \mathrm{~mm} \times 1 \mathrm{~mm} \times 1 \mathrm{~mm}$. Required tip and tilt angles for the alignment of lenses and mirrors had been specified as $\pm 0,3^{\circ}$.

For the kinematics of the six-axis manipulator a symmetric, hybrid serial-parallel structure has been chosen that consist of three inextensible struts which connect three non-collinear points of its platform to its base (Figure 3 ).

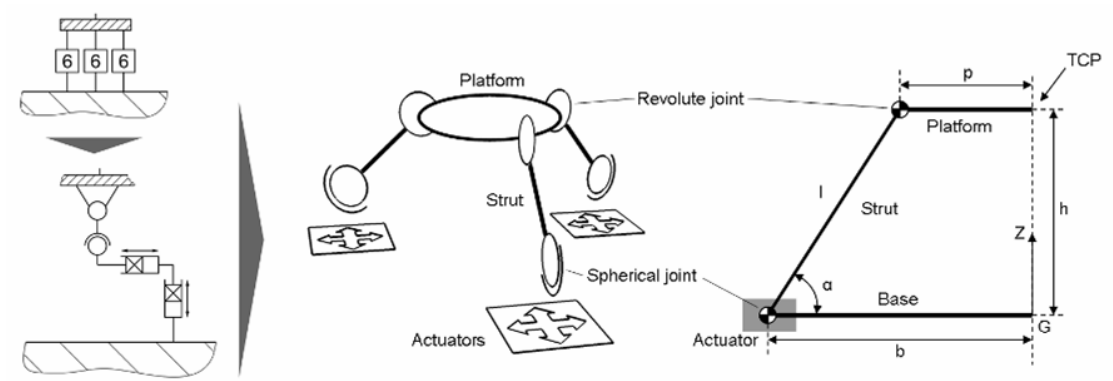

Fig. 3. Kinematical structure of the alignment module: three parallel kinematic chains with six DoF each (left top), consisting of an actuated serial x-y-tray, a spherical joint and a revolute joint (left bottom); base radius $b$, platform radius $p$, length of the struts $l$, strut angle $\alpha$, height $h$ 
The motion of the manipulator is obtained by moving the lower ends of the struts on the base plane by means of three identical x-y trays. Thereby, all the manipulator's actuators can be mounted on the base, achieving a higher payload capacity and smaller actuator sizes. Further advantages over other parallel kinematics like hexapods are a reduced collision risk of the struts due to the smaller number of joints and connecting elements, resulting in lower weight and higher accuracy [5].

As each strut of a symmetric 6-DoF parallel manipulator must have six degrees of freedom, it must contain four degrees of freedom in addition to the two of the actuated $x-y$ trays. Thus, a configuration of a 3-DoF spherical joint at the lower and a revolute joint at the upper end of each strut has been chosen to further reduce the moving masses and the influence of angle errors.

The orientation of the $x-y$-trays influences the dimensions of the manipulator, the actuator load as well as the work space geometry and size. Three possible arrangements are depicted in figure 4 where the table shows the normalized minimal dimensions of the manipulator base. For type 1 all trays are aligned with the $\mathrm{x}$ - and $\mathrm{y}$-axis of the base coordinate system. This leads to a minimal required space and a rectangular workspace in each horizontal section, while type 2 and 3 lead to workspaces with $120^{\circ}$ symmetries, in line with the kinematical design.

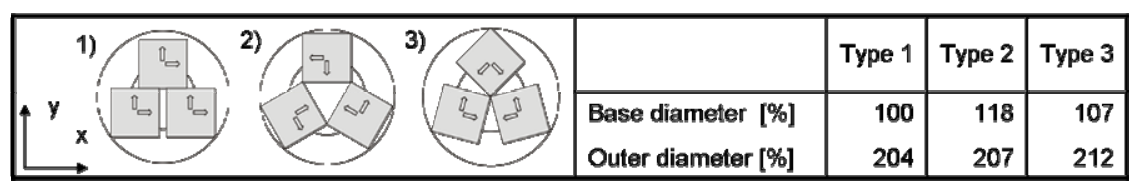

Fig. 4. Possible orientations of the $x-y$-trays

A vertical payload (z-axis) is most evenly distributed on the actuators for type 3 orientation, reducing the required actuating and blocking forces [Figure 5].

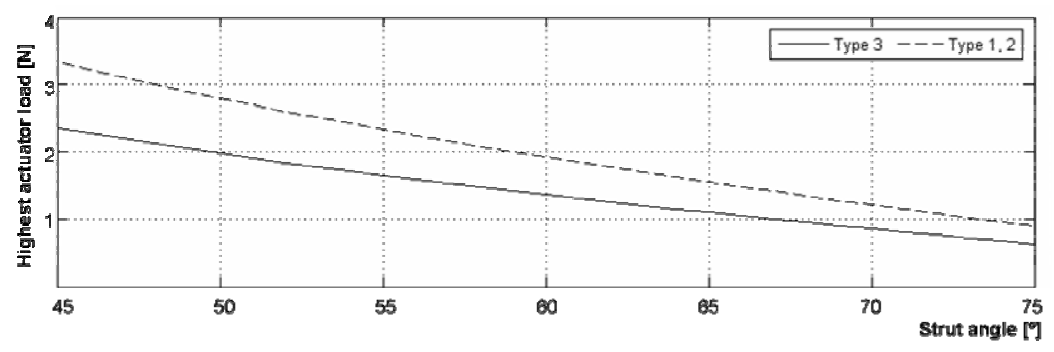

Fig. 5. Comparison of highest actuator loads $(b=50 \mathrm{~mm}, p=25 \mathrm{~mm}$, load $=1 \mathrm{~kg})$

For the $\mathrm{x}$-y-trays of the alignment module stacked piezo-based positioners with a travel of $\pm 5.5 \mathrm{~mm}$ and a step width of 50 to $500 \mathrm{~nm}$ have been chosen [6].

These linear positioners (SLC-1720) exhibit a very good ratio between outer dimensions and travel range with actuating forces above $2 \mathrm{~N}$, blocking forces above $3 \mathrm{~N}$ and allow for vertical loads of $40 \mathrm{~N}$. 
The resulting workspace of the kinematics are shown in figure 6 for the different types of actuator orientation. As mentioned above a symmetrical design of the manipulator leads to a workspace that is symmetrical to the y-z plane as shown.

For work space analysis the biggest possible cube inside the workspace is being identified, where the platform's zero position forms the center of the cube. Onedimensional comparisons are based on the edge length of this cube - called work space index. A calculation of work space indices for all actuator orientations shows an advantage for type 3 of $4,992 \mathrm{~mm}$ compared to $4,372 \mathrm{~mm}$ for type 1 and 2 .

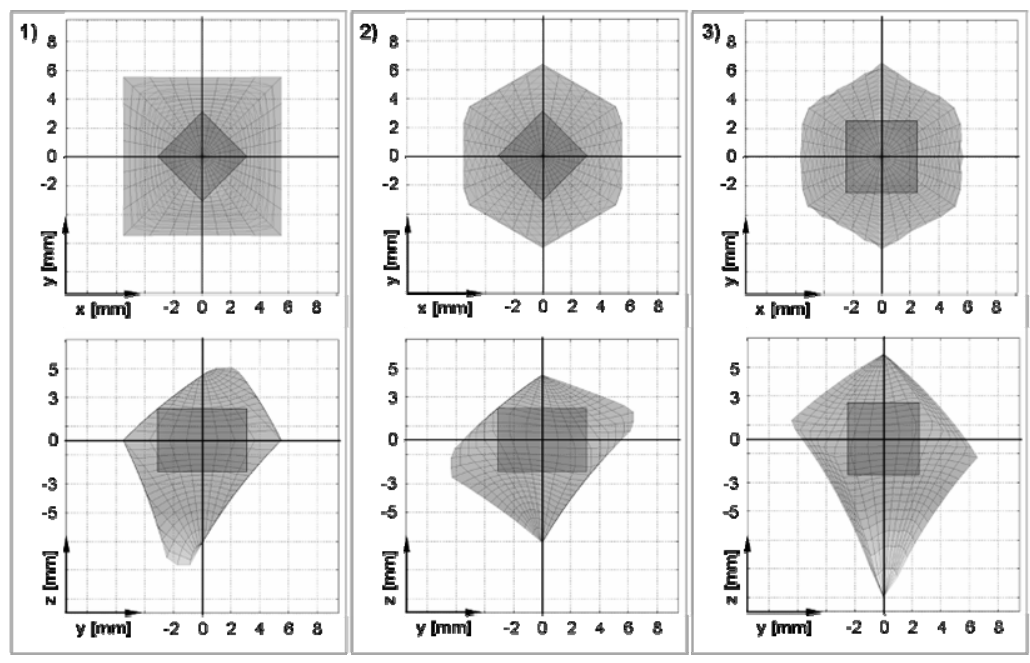

Fig. 6. Highest actuator loads $\left(b=50 \mathrm{~mm}, p=25 \mathrm{~mm}, \alpha=45^{\circ}\right)$

For the optimization of the geometrical dimensions, an analysis of the central stiffness matrix allows the derivation of following design guideline: If the platform radius is half the size of the base radius, the stiffness matrix will be diagonalized, i.e. the deformations induced by applied forces and moments are decoupled. Applied forces only cause translational, moments only rotational deformations [5].

Other aspects that have been analyzed and taken into account are the size and shape of the resulting workspace, the overall size of the system as well as the maximum deflection of the spherical and revolute joints. The latter aspect is of special relevance for the design of flexure-based mechanisms as the required range of motion is the decisive parameter for the dimensioning of flexure joints. Figure 7 shows an exemplary analysis of maximum joint deflection for the platform-sided revolute joints. The underlying calculations are based on tip-tilt movements around the $\mathrm{x}$ - and $y$-axis of $\pm 3^{\circ}$ for all positions within the workspace.

Figure 8 depicts the effect of changes in platform radius and strut angle on the size of the work space, represented by the introduced work space index. With a defined base radius the influence of the strut angle proves to be greater, as the transmission ratio of vertical platform travel (z-axis) to actuator travel is primarily depending on this parameter, i.e. the smaller the strut angle, the bigger the transmission ratio and thereby the larger the vertical travel range of the platform. 


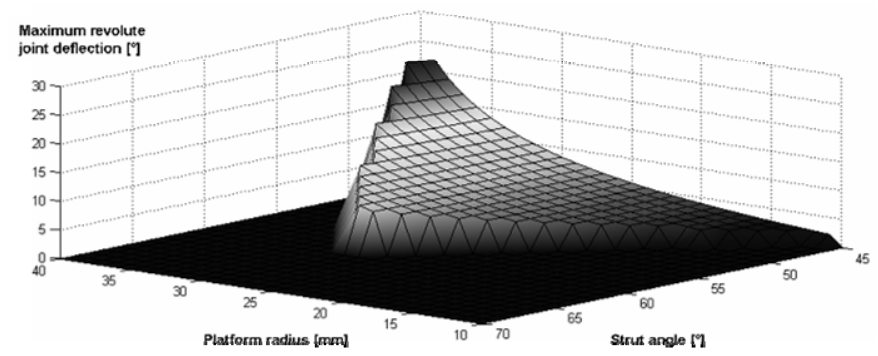

Fig. 7. Analysis of maximum joint deflections for platform sided revolute joints $(b=50 \mathrm{~mm}$, tip-tilt-movements around $x$ - and $y$-axis of $\pm 3^{\circ}$ for all positions within the work space; zero values are a result of workspace limits)

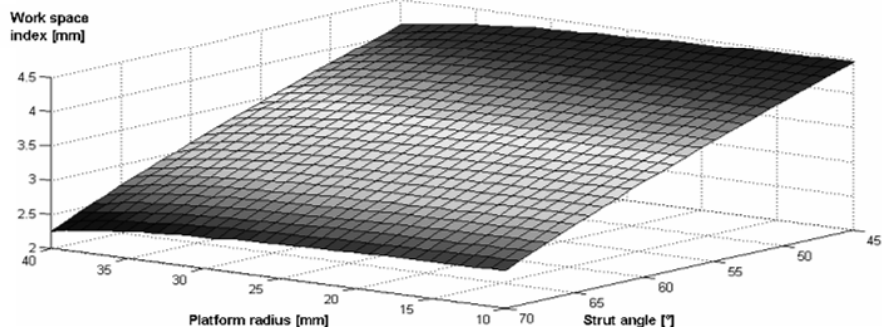

Fig. 8. Parameter study of strut angle and platform radius $(b=50 \mathrm{~mm})$

\section{Design and Dimensioning of the Flexure-Based Mechanism}

To maximize the accuracy of the alignment module a continuous monolithic construction has been realized by designing all joints as flexures to eliminate the presence of friction, wear and clearances.

The developed analysis tools have been applied to calculate the required joint displacements as $\pm 8-10^{\circ}$ for the tilt angles of the revolute and spherical joints and $\pm 12.5^{\circ}$ for the torsion angle around the strut axis. For such large displacements a special type of flexure design has been chosen that differs from the wide-spread and well-known notch-type joints or leaf springs. These two groups of flexures have been researched for years and are widely used for small-displacement mechanisms with high requirements on precision, but suffer from poor off-axis stiffness and significant axis drift for larger deformations.

The applied concept for a compliant revolute joint has been presented in a paper from Kota and Moon [7]. The proposed design generates pure rotational motion with widely reduced axis drift as well as a superior off-axis stiffness compared to many other types of flexure designs. Joint deflections, i.e. rotational movements, are based on the torsion of a cross-shaped beam which results in off-axis stiffness that is 220 to 38.000 times higher than the stiffness around the axis of motion. 
Based on this principle a miniaturized spherical joint has been designed for the manipulator. The three motion axes have been divided into two intersecting crossshaped beams for the tip and tilt movements of the struts and a third flexure element for the torsional degree of freedom. The platform-sided revolute joints have been based on the same concept (figure 9).
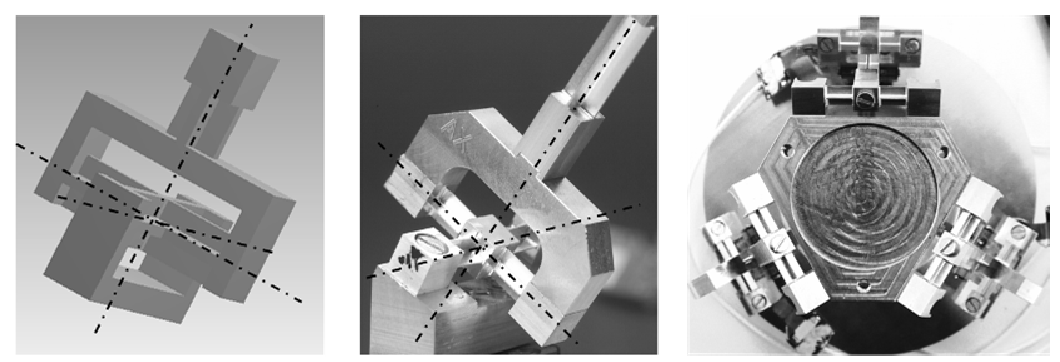

Fig. 9. Flexure design for spherical and revolute joints (left: concept of spherical joint; middle: spherical joint designed for and manufactured by micro milling; right: top view of alignment module showing the platform-sided revolute joints)

Based on the described design approach a first prototype of the manipulator has been set up. The calculated and simulated deformations of the flexures could be verified and measurements to characterize the accuracy and repeatability of the system have been conducted on a coordinate measurement machine (CMM).

The positioning accuracy of the manipulator could by means of kinematic calibration be improved from 50 micrometers down to 6 micrometers positioning accuracy within the biggest possible cube inside the workspace. An evaluation of the manipulator's repeatability showed that the measurements varied within the uncertainty of the CMM (around 0,75 micrometers). As the full potential of the design approach could not be verified by these measurements, first interferometer measurements have been conducted to characterize the motion resolution of the alignment module (figure 10).
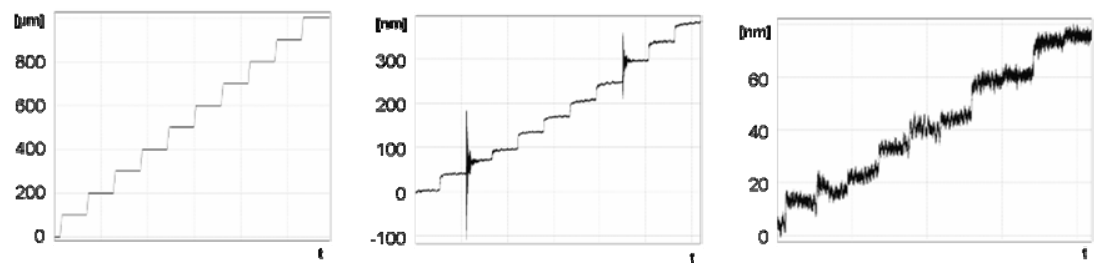

Fig. 10. Interferometer measurements to characterize the motion resolution (left: $100 \mu \mathrm{m}$ steps; middle: $50 \mathrm{~nm}$ steps; right: $10 \mathrm{~nm}$ steps; closed-loop control of the actuators)

These measurements proved sub-micron motion resolution and promise more reliable results for a further characterization of accuracy and repeatability. It can be seen that the flexure-based mechanical design of the alignment module allows a widely unaffected transformation of the actuators' motion onto the platform 


\section{Conclusion and Outlook}

Based on the requirements from producing miniaturized laser systems, a concept for a flexible robot-based precision assembly has been introduced that features a modular 6-axis alignment module. For a chosen hybrid kinematical structure different analyses for the dimensioning of a flexure-based mechanism have been presented, which led to the design of a prototypal alignment module.The described design approach makes use of an innovative concept for large-displacement compliant joints with superior qualities compared to other notch-type or leaf spring flexures. This concept has been adopted and extended to be applied for all spherical and revolute joints of the alignment module.

First measurement results from a CMM proved a repeatability of the manipulator below one micrometer, helped to improve the positioning accuracy significantly, but could not characterize the full potential of the system. Additional interferometer measurements regarding the achievable minimum step width showed far better results and will be continued for a more detailed characterisation of the alignment module.

\section{Acknowledgments}

The authors thank the »Deutsche Forschungsgemeinschaft (DFG)« for the support of the project within the Cluster of Excellence »Integrative Production Technologies for high-wage Countries« at RWTH Aachen.

\section{References}

1. Fatikow, S., Rembold, U.: Microsystem technology and microrobotics. Teubner, Stuttgart, Leipzig, p. 27 (2000)

2. Brenner, K.-H., Jahns, J.: Microoptics - From Technology to Applications, p. S. 34. Springer, New York (2004)

3. Tummala, R.: Fundamentals of microsystems packaging, New York, p. S. 18 (2001)

4. Loosen, P., Brecher, C., Schmitt, R.: Flexibel automatisierte Montage von Festkörperlasern, wt Werkstatttechnik online 11/12-2008, pp. 955-960 (2008)

5. Tahmasebi, F.: Kinematic Synthesis and Analysis of a Novel Class of Six-DOF Parallel Minimanipulators. Ph.D. Dissertation College Park: Univ. of Maryland (1993)

6. SmarAct GmbH, http: / / www. smaract. de

7. Kota, S., Moon, Y.-M., Trease, B.P.: Design of Large-Displacement Compliant Joints. Journal of Mechanical Deisgn, ASME 127, 788-798 (2005) 